\title{
MARROW STROMAL CELLS FOR CELLULAR CARDIOMYOPLASTY: FEASIBILITY AND POTENTIAL CLINICAL ADVANTAGES
}

Jih-Shiuan Wang, MD ${ }^{\mathrm{a}, \mathrm{b}}$

Dominique Shum-Tim, MD

Jacques Galipeau, $\mathrm{MD}, \mathrm{PhD}^{\mathrm{c}}$

Edgar Chedrawy, MD

Nicoletta Eliopoulos, $\mathrm{PhD}^{\mathrm{c}}$

Ray C.-J. Chiu, MD, $\mathrm{PhD}^{\mathrm{a}}$
Objectives: Marrow stromal cells are mesenchymal stem cells able to differentiate into cardiomyocytes in vitro. We tested the hypothesis that marrow stromal cells, when implanted into myocardium, can undergo milieu-dependent differentiation and express cardiomyogenic phenotypes in vivo.

Methods: Isogenic adult rats were used as donors and recipients to simulate autologous transplantation. Marrow stromal cells isolated from donor leg bones were culture-expanded, labeled with 4',6-diamidino-2-phenylindole, and then injected into the myocardium of the recipients. The hearts were harvested from 4 days to 12 weeks after implantation, and the implant sites were examined to identify the phenotypes of the labeled marrow stromal cells.

Results: Viable cells labeled with 4',6-diamidino-2-phenylindole can be identified in host myocardium at all time points after implantation. Implanted marrow stromal cells show the growth potential in a myocardial environment. After 4 weeks, donor cells derived from marrow stromal cells demonstrate myogenic differentiation with the expression of sarcomeric myosin heavy chain and organized contractile proteins. Positive staining for connexin 43 indicates the formation of gap junctions, which suggests that cells derived from marrow stromal cells, as well as native cardiomyocytes, are connected by intercalated disks.

Conclusions: Different cell sources have been used as donor cells for cellular cardiomyoplasty. Our findings indicate that marrow stromal cells can also be used as donor cells. In an appropriate microenvironment they will exhibit cardiomyogenic phenotypes and may replace native cardiomyocytes lost by necrosis or apoptosis. Because marrow stromal cells can be obtained repeatedly by bone marrow aspiration and expanded vastly in vitro before being implanted or used as autologous implants, and because their use does not call for immunosuppression, the clinical use of marrow stromal cells for cellular cardiomyoplasty appears to be most advantageous. (J Thorac Cardiovasc Surg 2000;120:999-1006)
From the Division of Cardiothoracic Surgery, McGill University, ${ }^{\mathrm{a}}$ the Division of Hematology Oncology, Jewish General Hospital/McGill University, ${ }^{\mathrm{c}}$ Montreal, Quebec, Canada, and the Division of Cardiovascular Surgery, Yang-Ming University/ Veterans General Hospital, ${ }^{\text {b }}$ Taipei, Taiwan.

Read at the Eightieth Annual Meeting of The American Association for Thoracic Surgery, Toronto, Ontario, Canada, April 30-May 3, 2000.

Received for publication May 11, 2000; revisions requested June 14, 2000; revisions received July 6, 2000; accepted for publication July 18, 2000.

Address for reprints: Ray C.-J. Chiu, MD, The Montreal General Hospital, 1650 Cedar Ave, Room C9-169, Montreal, Quebec, Canada H3G 1A4 (E-mail: rchiu@po-box.mcgill.ca).

Copyright (C) 2000 by The American Association for Thoracic Surgery

$0022-5223 / 2000 \$ 12.00+0 \quad \mathbf{1 2 / 6 / 1 1 0 2 5 0}$

doi:10.1067/mtc.2000.110250 iseases of the cardiovascular system remain a leadDing cause of morbidity and mortality worldwide. ${ }^{1}$ Congestive heart failure is the only cardiovascular disorder increasing in prevalence. ${ }^{2}$ Quantitative deficiency of cardiomyocytes and progressive ventricular remodeling characterize the final pathway of heart failure caused by various etiologies. ${ }^{3}$ Cellular cardiomyoplasty ${ }^{4}$ (CCM), in which one delivers appropriate donor cells into the injured myocardium, targets the basic pathophysiology of heart failure and represents a novel means of augmenting the myocyte number and the contractile function of a failing heart. Data derived from animal models have demonstrated the feasibility of this approach, ${ }^{4-8}$ but the optimal choice of donor cell source remains controversial. 
Bone marrow stromal cells (MSCs), which are mesenchymal stem cells, ${ }^{9}$ have been shown to have the potential of differentiating into cardiomyocytes in vitro when treated with 5 -azacytidine. ${ }^{10}$ Since autologous MSCs can be obtained from patients by simple routine bone marrow aspiration, and their use as donor cells does not require fetal tissue or immunosuppression, this approach appears to be highly advantageous for clinical use.

In this study, we tested the hypothesis that MSCs implanted into myocardium can undergo milieu-dependent differentiation, form long-term grafts expressing cardiomyogenic phenotypes, and be incorporated into the host myocardium to form a syncytium.

\section{Methods}

Animals. Male inbred Lewis rats (200 to $250 \mathrm{~g}$ ) were obtained from Charles River Laboratories (Laprairie Co, Quebec). These isogenic rats were used as donors and as recipients to simulate autologous implantation clinically. All animals received humane care in compliance with the "Guide for the Care and Use of Laboratory Animals," prepared by the Institute of Laboratory Animal Resources, National Research Council, and published by the National Academy Press (revised 1996), and the "Guide to the Care and Use of Experimental Animals" of the Canadian Council on Animal Care.

Isolation and culture of MSCs. Isolation and primary culture of MSCs from the femoral and tibial bones of donor rats were performed according to Caplan's method. ${ }^{11}$ In brief, after donor rats were killed with an overdose of pentobarbital (100 mg/kg given intraperitoneally, MTC Pharmaceuticals, Cambridge, Ontario, Canada), the femoral and tibial bones were collected for isolation of MSCs. Cells isolated from bone marrow in $10 \mathrm{~mL}$ of complete medium were then introduced into tissue culture dishes. Medium was completely replaced every 3 days, and the nonadherent cells were discarded. Cultured MSCs were observed under phase microscope to assess the level of expansion and to verify the morphology at each culture medium change. To prevent the MSCs from differentiating or slowing their rate of division, each primary culture was replated (first passage) to 3 new plates when the cell density within colonies became $80 \%$ to $90 \%$ confluent, approximately 2 weeks after seeding. The adherent cells were released from the dishes with $0.25 \%$ trypsin in $1 \mathrm{mmol} / \mathrm{L}$ sodium ethylenediaminetetraacetic acid (Gibco Laboratories, Grand Island, NY), split 1:3, and seeded onto fresh plates. After the twice-passaged cells became nearly confluent, they were harvested and used for the implantation experiments described below after being labeled with 4',6-diamidino-2-phenylindole (DAPI).

Medium. The cells were routinely cultured in complete medium consisting of Dulbecco's modified Eagle's medium containing selected lots of $10 \%$ fetal calf serum and antibiotics $(100 \mathrm{U} / \mathrm{mL}$ of penicillin $\mathrm{G}, 100 \mu \mathrm{g} / \mathrm{mL}$ of streptomycin, and $0.25 \mu \mathrm{g} / \mathrm{mL}$ of amphotericin B, from Gibco Laboratories) at $37^{\circ} \mathrm{C}$ in a humidified atmosphere of $5 \%$ carbon dioxide.

MSC labeling. Sterile DAPI stock solution (Sigma, St Louis, Mo) was added to the culture medium at a final concentration of $50 \mu \mathrm{g} / \mathrm{mL}$ on the day of implantation. The dye was allowed to remain in the culture dishes for at least 30 minutes. The cells were rinsed at least 6 times in Hanks balanced salt solution to remove all excess (unbound) DAPI. Marrow stromal cells were then collected (approximately $1 \times$ $10^{6}$ cells for one implantation) and resuspended in minimal volume of the serum-free Dulbecco's medium and stored on ice (less than 1 hour) until implantation into the myocardium.

Implantation of MSCs. Anesthesia was induced and maintained with isoflurane (Abbott Laboratories, St Laurent, Quebec, Canada). The recipient animals' lungs were intubated with an 18-gauge intravenous catheter and connected to a Harvard rodent ventilator (Harvard Apparatus Co, South Natick, Mass) to be ventilated at 85 breaths/min. The heart was exposed through a $1.5-\mathrm{cm}$ left thoracotomy incision. Under direct vision, the MSC suspension was injected into the left ventricular wall with a 28 -gauge needle. The implantation site was marked by $8-0$ polypropylene sutures. The thoracotomy was closed with 4-0 monofilament sutures. The muscle and skin layers were closed with 4-0 absorbable sutures, and the animals were returned to their cages.

Histology and immunohistochemistry. Rats were killed for the final experiments at the following intervals after the cell implantation surgery: 4 days $/ 4$ rats, 2 weeks $/ 2$ rats, 4 weeks $/ 4$ rats, 6 weeks/ 4 rats, and 12 weeks/2 rats. After overdose with pentobarbital, the hearts were exposed and injected with $100 \mathrm{~mL}$ of saline solution $(0.9 \%)$ through the posterior wall of the left ventricle, avoiding the transplant area, then perfusion-fixed with $2 \%$ paraformaldehyde in phosphatebuffered saline solution (PBS). The lateral wall of the left ventricle was isolated from the remainder of the heart and was either cryoembedded, after protection with $20 \%$ sucrose in PBS, or embedded in paraffin. Sections $6 \mu \mathrm{m}$ in thickness were mounted on a set of gelatin-coated glass slides to ensure different stains could be used on successive sections of tissue cut through the implantation area. One of the sections was mounted without stain, so the DAPI-labeled donor cells could be located and viewed with fluorescence microscopy. An adjacent section was stained with hematoxylin and eosin to depict nuclei, cytoplasm, and connective tissue. Other serial sections were selected for immunostaining of sarcomeric myosin heavy chain molecules with MF20 (Developmental Studies Hybridoma Bank, University of Iowa, Iowa City, Iowa) or connexin 43 with rabbit polyclonal antibody (Zymed Laboratories Inc, San Francisco, Calif). These sections were incubated in primary antibodies overnight at $4^{\circ} \mathrm{C}$. For detection, we used secondary antibodies conjugated to Texas Red for MF20 and fluorescein for anti-connexin 43 (Vector Laboratories, Inc, Burlingame, Calif). Combined "DAPI and Texas Red" or "DAPI and fluorescein" in images were made by using a simultaneous excitation filter under reflected light fluorescence microscopy (BX-FLA; Olympus America, Inc, 


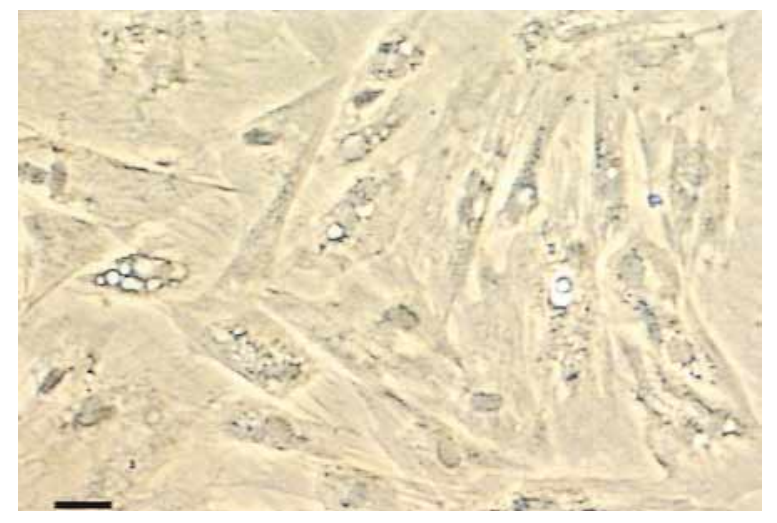

Fig 1. The morphology of rat MSCs in in vitro culture. Phase contrast photomicrograph of twice-passaged culture of MSCs just before implantation. Most adherent MSCs are practically fibroblastic in morphology. Scale bar represents $60 \mu \mathrm{m}$.

Huntington Station, NY). Digital images, transferred to a computer equipped with Image Pro software (Media Cybernetics, Silver Spring, Md), were subsequently printed (Stylus Photo 700, Epson, Long Beach, Calif) for publication.

\section{Results}

Cultured MSCs were observed under phase microscopy to assess the magnitude of expansion and to verify the morphology at each culture medium change. Most of the hematopoietic stem cells were not adherent to the culture plate and were removed with changes of medium. The adherent cells were seen as individual cells or colonies of only a few cells on day 6; however, they replicated rapidly and formed colonies of up to 100 cells after the first week of culture. By the end of the second week, the colonies of adherent cells had expanded in size, with each colony containing several hundred to several thousand cells. Adherent MSCs obtained from rat leg bone marrows had similar morphology, most being fibroblastic, with a few adipocytic polygonal cells (Fig 1). This phenotype was retained throughout repeated passages under nonstimulating conditions. Some adherent round cells were present but normally accounted for less that $5 \%$ of the adherent cell populations at the time cultures were ready to be replated on first passage, and these cells further reduced in frequency at the end of first and second passages. Since 3 new plates were generated by the replating of each near-confluent plate, a single primary culture generated 3 plates at first passage and 9 plates at second passage. Since each near-confluent plate contained on average 1.0 million cells, a single primary

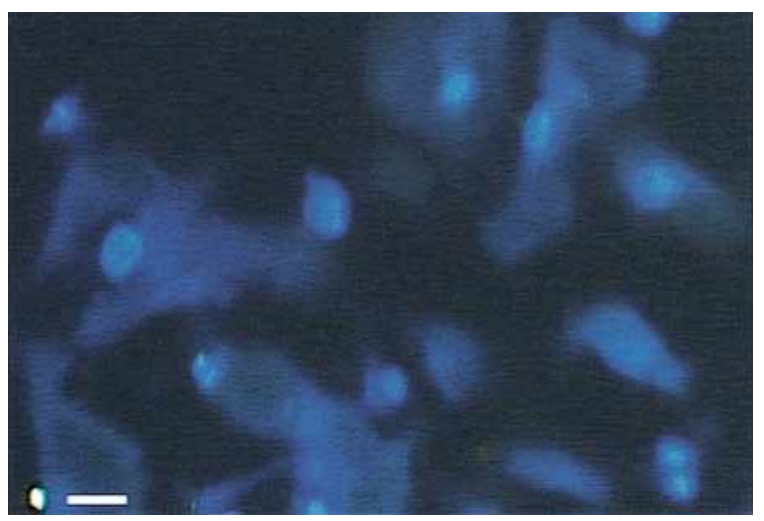

Fig 2. Labeling of rat MSCs in vitro before implantation into the recipient rat hearts. DAPI epifluorescence (blue fluorescence) of twice-passaged culture of MSCs. Note the obvious nuclear and faint cytoplasmic blue fluorescence. Virtually $100 \%$ of the cultured cells are labeled by DAPI. Scale bar represents $30 \mu \mathrm{m}$.

culture starting with 100 to 500 adherent MSCs could be expanded to well over 9.0 million cells by the second passage. We labeled twice-passaged MSCs with DAPI just before implantation surgery. The DAPIlabeled MSCs showed clear nuclear and faint cytoplasmic blue fluorescence when viewed under an epifluorescence microscope (Fig 2). Virtually $100 \%$ of the cultured cells were labeled by DAPI.

Implantation mortality was zero after a period of practice. All recipient rats survived to the time they were killed. Gross examination of the specimen did not reveal any structural abnormalities except sometimes a tiny fibrotic change around implantation puncture sites (marked by 8-0 suture). Paraffin sections as well as frozen sections (for immunohistochemistry) of the implant sites of the hearts were made to evaluate the morphology and phenotype changes of implanted MSCs. DAPI-labeled cells could be identified in all specimens except 2 rats (one 6 weeks, the other 12 weeks) at all time points to at least 12 weeks after surgery, the latest date assayed.

Four days after implantation numerous scattered DAPI-labeled cells (blue fluorescence) were found in the specimen (Fig 3, A). Hematoxylin and eosin staining of the consecutive section showed concordance between dense hematoxylin staining and presence of DAPI epifluorescence (Fig 3, B). The MSC-derived donor cells showed an immature appearance with a large nucleus-to-cytoplasm ratio. There was no obvious inflammatory response at this time. Four weeks after implantation, a few clearly DAPI-labeled cells were found to be incorporated into the host myocardium (Fig 

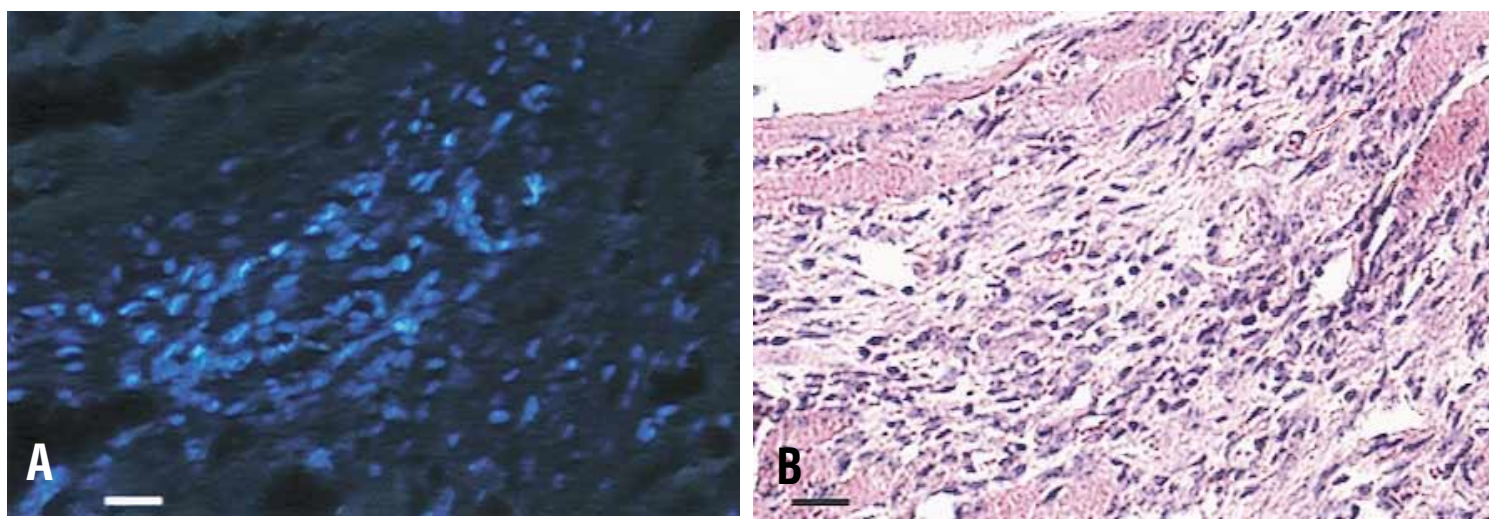

Fig 3. MSC grafts in isogenic rat recipient hearts 4 days after implantation. A, DAPI epifluorescence (blue fluorescence) photomicrographs. Note the numerous scattered DAPI-labeled cells present in this field. B, Hematoxylin and eosin stain. Consecutive section to that shown in A. Note concordance between dense hematoxylin staining and presence of DAPI epifluorescence. The labeled MSC-derived donor cells show immature appearance with large nucleus-to-cytoplasm ratio. Scale bars represent $30 \mu \mathrm{m}$.
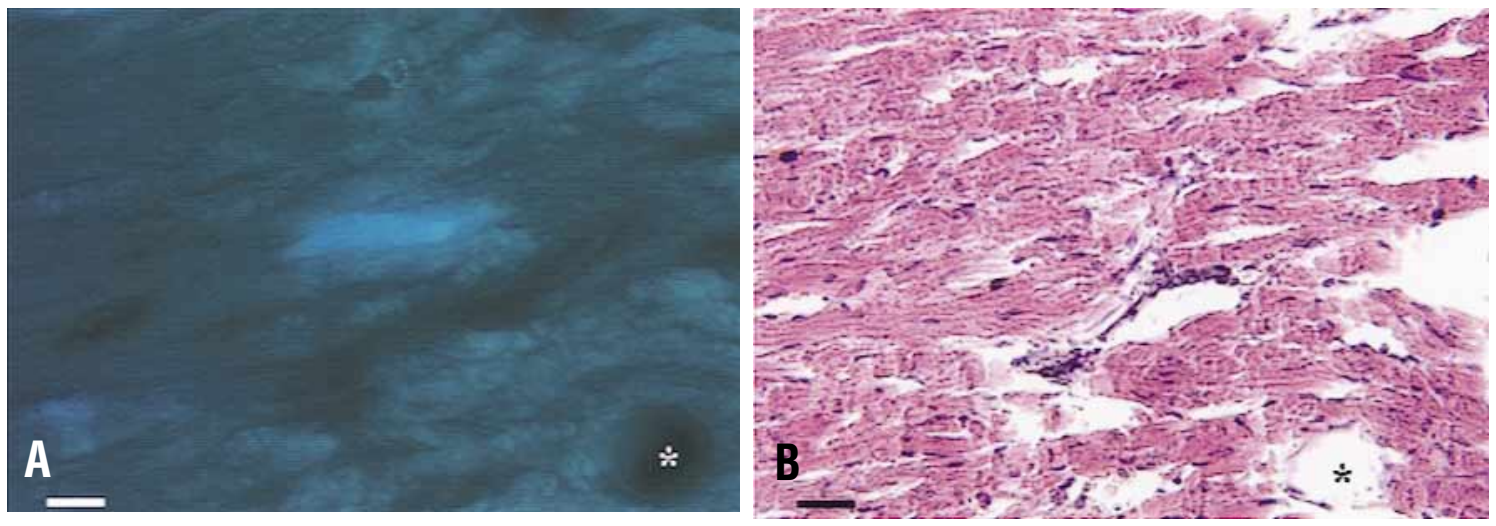

Fig 4. MSC grafts in isogenic rat recipient hearts 4 weeks after implantation. A, DAPI epifluorescence (blue fluorescence) photomicrographs. Incorporation of DAPI-labeled cells with the host myocardium. B, Hematoxylin and eosin stain. Consecutive section to that shown in A. Labeled MSC-derived cells have the same structure with that of the surrounding cardiomyocytes. Asterisks show corresponding cross section of a blood vessel in this pair of images (A and B). Scale bars represent $30 \mu \mathrm{m}$.

4, A). They had abundant cytoplasm and had aligned themselves with nonlabeled cells (host cardiomyocytes). Hematoxylin and eosin stain of consecutive section (Fig 4,B) showed that MSC-derived cells had the same histologic appearance as those of surrounding cardiomyocytes.

The phenotypes of implanted MSCs were confirmed by means of immunohistochemistry. Fig 5 is taken from a section of a heart 6 weeks after MSC implantation. We used simultaneous excitation filter to combine DAPI (blue fluorescence) and Texas Red (red fluorescence) images of the same microscopic field. DAPI-labeled cells (ie, MSC-derived) have centrally located nuclei and were stained positive for sarcomeric myosin heavychain molecules (with use of MF20 primary antibody and Texas Red conjugated secondary antibody). The surrounding host myocardium (nonlabeled cells) also stained positive. These positively stained cells (MSCderived and host) had clear striations, which represent organized contractile proteins. Again, DAPI-labeled donor cells aligned in parallel with nonlabeled myocytes (host cardiomyocytes) and were incorporated into host myocardium. To further confirm the syncytial incorporation of implanted MSCs with host cardiomy- 


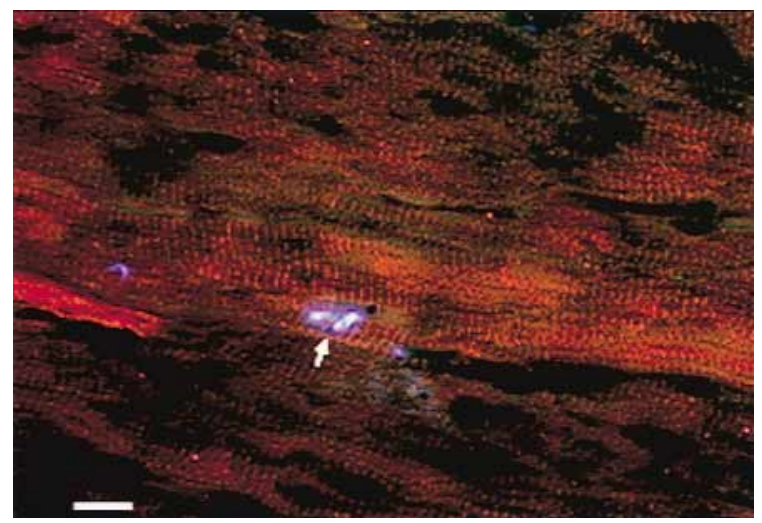

Fig 5. Expression of sarcomeric myosin heavy-chain molecules of MSC-derived donor cells in specimens taken 6 weeks after implantation. Combined DAPI (blue fluorescence) and Texas Red (red fluorescence) images of the same microscopic field. Arrow shows DAPI-labeled cell is positively stained with sarcomeric myosin heavy-chain immunofluorescence (with use of MF20 primary antibody and Texas Red conjugated secondary antibody), which shows clear striations. Scale bar represents $15 \mu \mathrm{m}$.

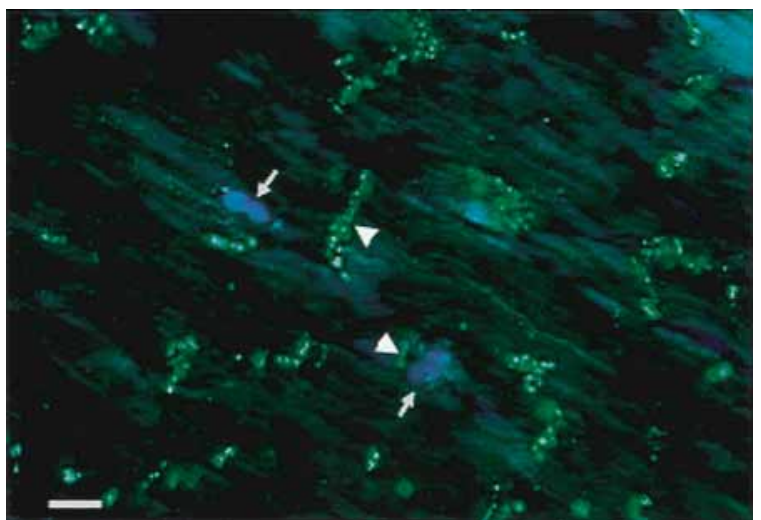

Fig 6. Expression of connexin 43 of MSC-derived donor cells in a specimen 6 weeks after implantation. Combined DAPI and fluorescein (green fluorescence) image. Anti-connexin 43 immunofluorescence is elicited, by using fluorescein conjugated secondary antibody. Positive connexin 43 staining (arrowheads) is found at the interfaces between 1 DAPIlabeled cell (arrow) and neighboring non-labeled cells (host cardiomyocytes) and between non-labeled cells. Scale bar represents $15 \mu \mathrm{m}$.

Table I. Major concerns about cell source in clinical application of cellular cardiomyoplasty

\begin{tabular}{lcccc}
\hline Cell source & Ethical problem & Acquirement concern & Rejection & Potential oncogenicity \\
\hline Fetal cardiomyocytes & Yes & Yes & Yes & No \\
Adult cardiomyocytes & No & Yes & No & Yes \\
Skeletal myoblasts & No & Yes & Yes & No \\
Cell line cells & No & No & Yes & Yes \\
Embryonic stem cells & Yes & Yes & No & Yes \\
Marrow stromal cells & No & No & No
\end{tabular}

Acquirement concern includes logistic and other difficulties in getting donor cells, potentially irreversible injury to recipient/donor, and the difficulty in cultivating and expanding cells in vitro.

ocytes, and to elucidate the nature of the cell junction, frozen sections were stained for connexin 43 , one of gap junction proteins in the intercalated disks, by using fluorescein conjugated secondary antibody. Again, by using a simultaneous excitation filter, an image of combined DAPI and fluorescein epifluorescence was obtained from a section taken 6 weeks after the implantation (Fig 6). Positive connexin 43 staining was found at the interfaces of DAPI-labeled cells (MSC-derived) and neighboring nonlabeled cells (host cardiomyocytes), and between nonlabeled cells. The green fluorescence of connexin 43 staining present along the axial borders of the cells also showed the alignment of the engrafted cells with surrounding cardiac myofibers.

\section{Discussion}

Deficiency of cardiomyocytes in different types of heart failure may result from necrosis or from apoptosis. ${ }^{12}$ Myocardial regeneration and repair after injury is severely limited by the inability of postembryonic heart tissue to recruit muscle progenitor cells. Although terminally differentiated cardiomyocytes have shown some evidence of mitotic division in the adult heart, ${ }^{13}$ the rate of proliferation is minute and cannot meet the demand for tissue regeneration. Therefore, efforts to develop strategies to repair the injured myocardium for treatment of heart failure are both rational and useful. CCM, which uses the technique of cell transplantation for myocardial regeneration, aims to replenish myocyte 
number and thus represents a new strategy for treatment of heart failure.

Different donor cell sources have been used experimentally for CCM, and some investigators have reported that CCM could improve the function of impaired ventricle. ${ }^{6,7}$ However, each donor cell source, such as fetal cardiomyocytes or skeletal myoblasts, appears to encounter various ethical, biologic, or technical limitations that must be overcome if it is to be considered for application to human patients (Table I). MSCs are the second group of stem cells, other than hematopoietic stem cells in the bone marrow, with the capacity to differentiate into cells that can roughly be defined as mesenchyma. Thus, they are also referred to as mesenchymal stem cells. ${ }^{14}$ Under controlled in vitro conditions, MSCs have been reproducibly guided to multiple mesenchymal lineages, including those of bone, cartilage, adipocytes, myocytes, and even cardiomyocytes. ${ }^{10,15}$ We hypothesized that the myocardial microenvironment would supply the proper conditions and signals for the cardiomyogenic differentiation of MSCs. Thus, transplanting MSCs into myocardium would induce them through milieu-dependent differentiation to develop into cardiomyocytes.

In this study, rat MSCs showed a capacity to proliferate in culture with an attached well-spread morphology (Fig 1). We generated a morphologically homogeneous phenotype population by serial passage of the cells composing the adherent layer, and we removed most of the hematopoietic stem cells using this method. We successfully expanded MSCs from 500 to 9 million per rat by about 14 cell doublings before implantation. This feature holds great significance for clinical application of CCM in terms of the number of cells needed to improve cardiac function. ${ }^{16}$ In addition to the fact that bone marrow aspiration is a simple and routine clinical activity, this ability to cultivate and vastly multiply MSCs in vitro should encourage the clinical use of this cell source.

Experimentally, so the fate of the implanted cells may be traced, MSCs are labeled while in culture with DAPI. DAPI, a fluorescent dye, has been used as a cell marker in study of cell biology. ${ }^{17}$ It labels the nucleus of a cell because of a high affinity for dsDNA. Its mechanism of action is that it forms strong electrostatic interactions with adenine-thymine-rich regions of DNA. The fluorescence quantum yield of the free dye is very low, but the binding to DNA results in a highly fluorescent complex. ${ }^{18}$ The other cell components to which DAPI binds to form fluorescent complexes are the protein tubulin and microtubules. ${ }^{19}$ DAPI is nontoxic to living cells and does not alter the ultrastructure of cell organelles. To further confirm the reliability of this technique, we performed characterization in vitro followed by in vivo analysis. Under the labeling concentration of $50 \mu \mathrm{g} / \mathrm{mL}$ for 30 minutes in culture, the labeled cells show clear nuclear and faint cytoplasmic staining and labeling efficiency is almost $100 \%$ (Fig 2). There is no DAPI fluorescence detectable in the culture medium. Our in vivo control studies, including injections into the myocardium of free DAPI solutions, unlabeled MSCs or lysed DAPI-labeled MSCs while performing the same epifluorescence read-out, confirmed the specificity of this cell labeling technique for our study (data not shown).

Recently, Makino and associates ${ }^{10}$ could identify a single clone of adherent fibroblast-like cells that when treated with 5-azacytidine would differentiate into cells with some morphologic features of cardiac muscle and expression of cardiac-specific genes. Tomita and colleagues ${ }^{20}$ transplanted fresh, cultured, and 5-azacytidine-treated bone marrow cells into the cryoinjury scar in a rat model and reported cardiomyogenic differentiation with expression of cardiac specific protein, troponin I. Our results further demonstrated that the myocardial microenvironment could support the growth and induce the cardiomyogenic differentiation of MSCs after their implantation. The mechanisms and the signals to induce MSC-derived donor cells to undergo cardiomyogenic differentiation within the myocardial environment are not fully understood. Recent studies have led to the suggestion that the MSCs may act as cycling stem cells and that they are involved in the mesengenic process for self-maintenance and repair of different mesenchymal tissues after birth. ${ }^{21}$ Moreover, the progeny of MSCs expressed genes in a tissue-specific manner according to the destination to which they arrived. ${ }^{22}$ This homing ability and the ability to acquire the phenotypes of different target tissues suggest the microenvironment plays a significant role on the differentiation of these cells. On the basis of recent studies on stem cell biology and differentiation, it is assumed that specific molecules, growth factors, cytokines, and interactions among stem cells, host cells, and extracellular matrix are necessary, and they are presented to the stem cells in a temporal and sequential manner in order to induce the expression of related developmental genes in sequence and driving their differentiation into different tissues. ${ }^{23,24}$ We suggest that the normal myocardial microenvironment may supply the proper mix and sequential exposure to the cardiomyogenic specific growth factors and differentiation molecules, so that the implanted MSCs could develop to fully mature cardiomyocytes. 
We also demonstrated that these MSC-derived donor cells are not only myogenically differentiated but also developed mature organized contractile protein. In addition, they connected and aligned with host cardiomyocytes by gap junctions. The intact heart is a syncytium with an elaborate three-dimensional myocardial orientation and specialized cell junctions. ${ }^{25}$ This organization ensures the orderly propagation of electrical signals and the coordinated contraction of the myocardium. In CCM, implanted cells are used to perform specific physiologic roles, and thus the phenotypes and the maturation of the cells and their spatial location in relation to the host myocardial supracellular organization are critical to their application. These 2 characteristics of MSC-derived donor cells found in this study are prerequisites for implanted donor cells to improve the contractile function of recipient hearts.

We appreciate the technical assistance of Mrs Minh Duong, BSc. The MF20 antibody, developed by Donald A. Fischman, MD, was obtained from the Developmental Studies Hybridoma Bank, which was developed under the auspices of the NICHD and is maintained by The University of Iowa, Department of Biological Sciences, Iowa City, Iowa.

\section{REFERENCES}

1. L'enfant C. NHLBI at 50: reflections on a half-century of research on the heart, lungs, and blood. National Heart, Lung, and Blood Institute. JAMA 1998;280:2062-4.

2. Schocken DD, Arrieta MI, Leaverton PE, Ross EA. Prevalence and mortality rate of congestive heart failure in the United States. J Am Coll Cardiol 1992;20:301-6.

3. Li P, Zhang X, Capasso JM, Meggs LG, Sonnenblick EH, Anversa P. Myocyte loss and left ventricular failure characterize the long-term effects of coronary artery narrowing or renal hypertension in rats. Cardiovasc Res 1993;27:1066-75.

4. Chiu RCJ, Zibaitis A, Kao RL. Cellular cardiomyoplasty: myocardial regeneration with satellite cell implantation. Ann Thorac Surg 1995;60:12-8.

5. Soonpaa MH, Koh GY, Klug MG, Field LJ. Formation of nascent intercalated disks between grafted fetal cardiomyocytes and host myocardium. Science 1994;264:98-101.

6. Li RK, Jia ZQ, Weisel RD, Mickle DAG, Zhang J, Mohabeer MK, et al. Cardiomyocytes transplantation improves heart function. Ann Thorac Surg 1996;62:654-61.

7. Taylor DA, Atkins BZ, Hungspreugs P, Jones TR, Reedy MC, Hutcheson KA, et al. Regenerating functional myocardium: Improved performance after skeletal myoblast transplantation. Nature Med 1998;4:929-33.

8. Klug MG, Soonpaa MH, Koh GY, Field LJ. Genetically selected cardiomyocytes from differentiating embryonic stem cells form stable intracardiac grafts. J Clin Invest 1996;98:216-24.

9. Caplan AI. Mesenchymal stem cells. J Orthop Res 1991;9:64150.

10. Makino S, Fukuda K, Miyoshi S, Konishi F, Kodama H, Pan J, et al. Cardiomyocytes can be generated from marrow stromal cells in vitro. J Clin Invest 1999;103:697-705.
11. Wakitani S, Saito T, Caplan AI. Myogenic cells derived from rat bone marrow mesenchymal stem cells exposed to 5-azacytidine. Muscle Nerve 1995;18:1417-26.

12. Yeh ETH. Life and death in the cardiovascular system. Circulation 1997;95:782-6.

13. Kajstura J, Leri A, Finato N, Di Loreto C, Beltrami CA, Anversa P. Myocardial proliferation in end-stage cardiac failure in humans. Proc Natl Acad Sci U S A 1998;95:8801-5.

14. Prockop DJ. Marrow stromal cells as stem cells for nonhematopoietic tissues. Science 1997;276:71-4.

15. Pittenger MF, Mackay AM, Beck SC, Jaiswal RK, Douglas R, Mosca JD et al. Multilineage potential of adult human mesenchymal stem cells. Science 1999;284:143-7.

16. Williams RS. Cell cycle control in the terminally differentiated myocyte. Cardiol Clin 1998;16:739-48.

17. Kapuscinski J. DAPI: a DNA-specific fluorescent probe. Biotech Histochem 1995;70:220-33.

18. Tarnowski BI, Spinale FG, Nicholson JH. DAPI as a useful stain for nuclear quantitation. Biotech Histochem 1991;66:296-302.

19. Bonne D, Heusele C, Simon C, Pantaloni D. 4',6-diamidino-2phenylindole, a fluorescent probe for tubulin and microtubules. $\mathrm{J}$ Biol Chem 1985;260:2819-25.

20. Tomita S, Li RK, Weisel RD, Mickle DAG, Kim EJ, Sakai T, et al. Autologous transplantation of bone marrow cells improves damaged heart function. Circulation 1999;100(Suppl):II-247-56.

21. Caplan AI. The mesengenic process. Clin Plast Surg 1994;21:429-35

22. Pereira RF, Halford KW, O'Hara MD, Leeper DB, Sokolov BP, Pollard MD, et al. Cultured adherent cells from marrow can serve as long-lasting precursor cells for bone, cartilage, and lung in irradiated mice. Proc Natl Acad Sci U S A 1995;92:4857-61.

23. Fishman MC, Chien KR. Fashioning the vertebrate heart: earliest embryonic decisions. Development 1997;124:2099-117.

24. Smith AG, Henth JK, Donaldson DD, Wong GG, Moreau J, Stahl $\mathrm{M}$, et al. Inhibition of pluripotential embryonic stem cell differentiation by purified polypeptides. Nature 1988;336:688-90.

25. Burkitt HG. Wheater's functional histology: a text and color atlas. 3rd ed. New York: Churchill Livingstone; 1993. p. 93-111.

26. Li RK, Weisel RD, Mickle DAG, Jia ZQ, Kim EJ, Sakai T, et al. Autologous porcine heart cell transplantation improved heart function after a myocardial infarction. J Thorac Cardiovasc Surg 2000;119:62-8.

27. Koh GY, Klug MG, Soonpaa MH, Field LJ. Differentiation and long-term survival of $\mathrm{C} 2 \mathrm{C} 12$ myoblast grafts in heart. J Clin Invest 1993;92:1548-54.

28. Robinson SW, Cho PW, Levitsky HI, Olson JL, Hruban RH, Acker MA, et al. Arterial delivery of genetically labeled skeletal myoblasts to the murine heart: long-term survival and phenotypic modification of implanted myoblasts. Cell Transplant 1996;5:77-91.

\section{Discussion}

Dr Shinji Tomita (Toronto, Ontario, Canada). We are also involved in cell transplantation, especially using bone marrow. ${ }^{1}$ I want to ask Dr Wang several questions. First, what percentage of the transplanted cells are going to differentiate into cardiomyogenic cells? Next, what is the possible factor regarding the cardiac milieu? Is that like pressure or growth factor? 


\section{REFERENCE}

1. Tomita S, Li R-K, Weisel RD, Mickle DAG, Jia Z-Q, Kim EJ, et al. Autologous transplantation of bone marrow cells improves heart function. Circulation 1999;100(Suppl):II-247-56.

Dr Wang. Thank you for your question. We did not calculate the percentage of the cells that change to cardiomyocytes, although we did find that DAPI-labeled cells decreased significantly after 4 weeks in our specimen.

As to the other question, about the milieu-dependent differentiation, intuitively we think the myocardial environment may supply the proper mix and the sequential exposure of cardiomyogenic specific growth factor and differentiation molecules to the implanted marrow stromal cells. So we also think that a microenvironment is very important. We are now focusing on the microenvironment issue. We are trying to evaluate the impact of different microenvironments on these cells' differentiation.

Dr Yasuharu Noishiki (Yokohama, Japan). I have a different opinion related to the marrow cell transplantation. Because marrow cells are very young and primitive, they want to survive when they are transplanted into a different environment. When we transplant marrow cells, we can see the many angiogenic growth factors synthesized around the cells. So we can observe capillary ingrowth around the site. Do you have any data similar to these?

Dr Wang. Thank you for your question. We have no data now about angiogenesis, but for cellular cardiomyoplasty we still need to answer some issues-for example, if these cells can engraft in the ischemic myocardium or myocardial scar.

The other question is if the cellular cardiomyoplasty itself can induce angiogenesis. We have no data for this now. 